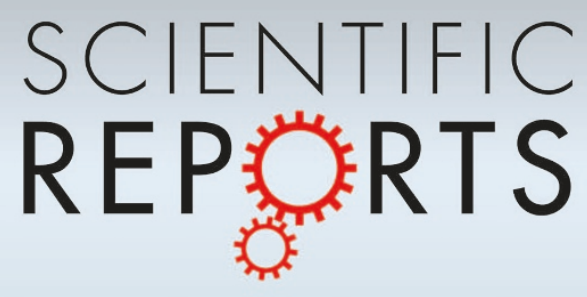

\title{
OPEN Magneto-optical fingerprints of distinct graphene multilayers using the giant infrared Kerr effect
}

SUBJECT AREAS:

MAGNETO-OPTICS

ELECTRONIC PROPERTIES AND

MATERIALS

Received

17 October 2013

Accepted

21 October 2013

Published

5 November 2013

Correspondence and requests for materials should be addressed to J.C. (Jcerne@buffalo.

\author{
Chase T. Ellis', Andreas V. Stier', Myoung-Hwan Kim', Joseph G. Tischler², Evan R. Glaser², \\ Rachael L. Myers-Ward ${ }^{2}$, Joseph L. Tedesco ${ }^{2,3}$, Charles R. Eddy Jr², D. Kurt Gaskill ${ }^{2} \&$ John Cerne ${ }^{1}$
}

'Department of Physics, University at Buffalo, SUNY, Buffalo, New York, USA, ${ }^{2}$ Electronics Science \& Technology Division Code 6800 , U.S. Naval Research Laboratory, Washington, DC, USA, ${ }^{3}$ American Society for Engineering Education, 1818 N Street NW, Washington, DC, USA.

The remarkable electronic properties of graphene strongly depend on the thickness and geometry of graphene stacks. This wide range of electronic tunability is of fundamental interest and has many applications in newly proposed devices. Using the mid-infrared, magneto-optical Kerr effect, we detect and identify over 18 interband cyclotron resonances (CR) that are associated with $A B A$ and $A B C$ stacked multilayers as well as monolayers that coexist in graphene that is epitaxially grown on $4 \mathrm{H}-\mathrm{SiC}$. Moreover, the magnetic field and photon energy dependence of these features enable us to explore the band structure, electron-hole band asymmetries, and mechanisms that activate a CR response in the Kerr effect for various multilayers that coexist in a single sample. Surprisingly, we find that the magnitude of monolayer Kerr effect CRs is not temperature dependent. This unexpected result reveals new questions about the underlying physics that makes such an effect possible.

T

he discovery of a stable monolayer flake of graphene by Novoselov et al. ${ }^{1}$ set off a massive investigation of the remarkable properties of mono- and multilayer graphene. The simple hexagonal carbon lattice of monolayer graphene results in a unique linear dispersion with low energy excitations that are massless and relativistic. From these characteristics arise fascinating properties such as square root magnetic field $(B)$ dependence of the cyclotron resonance (CR) frequency, a lowest Landau level (LL) independent of $B$, anomalous chiral quantum Hall effects ${ }^{2}$, unusual magnetic field and Fermi energy dependence of the AC Hall conductivity ${ }^{3}$, and the presence of a finite longitudinal conductivity $\sigma_{x x}$ without charge carriers ${ }^{4}$. Studies of multilayer graphene have proven equally interesting due to the existence of massive Dirac fermions, unique broken symmetry states ${ }^{5}$, and the existence of a readily tunable bandgap ${ }^{6-8}$. In addition, the electronic structure of multilayer graphene is highly dependent upon the stacking geometry and thickness of layers, yielding a wide range of electronic tunability for graphene systems ${ }^{17,18,24}$. To that end, both scientists and engineers alike have been fascinated by graphene and all of its possible applications, such as high frequency analog ballistic transistors ${ }^{8-10}$, surface enhanced spectroscopy $y^{2-4}$, photo-detectors ${ }^{11}$, transparent conductors ${ }^{13}$, and as proposed in this Report, ultra-fast optical modulators and tunable polarizers.

When placed in an out-of-plane magnetic field, multilayer graphene's electronic bands condense into discrete energy states (LLs) that are especially sensitive to the coupling strength between layers. This coupling strength is dependent upon the relative orientation of layers and the total number of layers in a graphene stack. Graphene's strong dependence on interlayer coupling has been demonstrated many times for monolayer ${ }^{5-7}$ (uncoupled) and bilayer $^{7-9}$ (coupled) graphene, which yield LL energies proportional to $\sqrt{B}$ and $B$, respectively. In this study we systematically investigate the effect of more complicated coupling schemes that are obtained from thicker graphene multilayers with various stacking geometries. This is especially of interest since studies suggest that interlayer coupling may play a role in producing asymmetries between the conduction and valence band energies, where band asymmetry has been attributed to the twisting of adjacent layers ${ }^{10}$ and next nearest neighbor interactions $^{11}$ (trigonal warping effects). However, experiments have also shown that metal electrical contacts on graphene ${ }^{12,13}$ may be responsible for band asymmetry, which is consistent with the absence of an observable band asymmetry in unprocessed graphene samples ${ }^{11,14,15}$. 

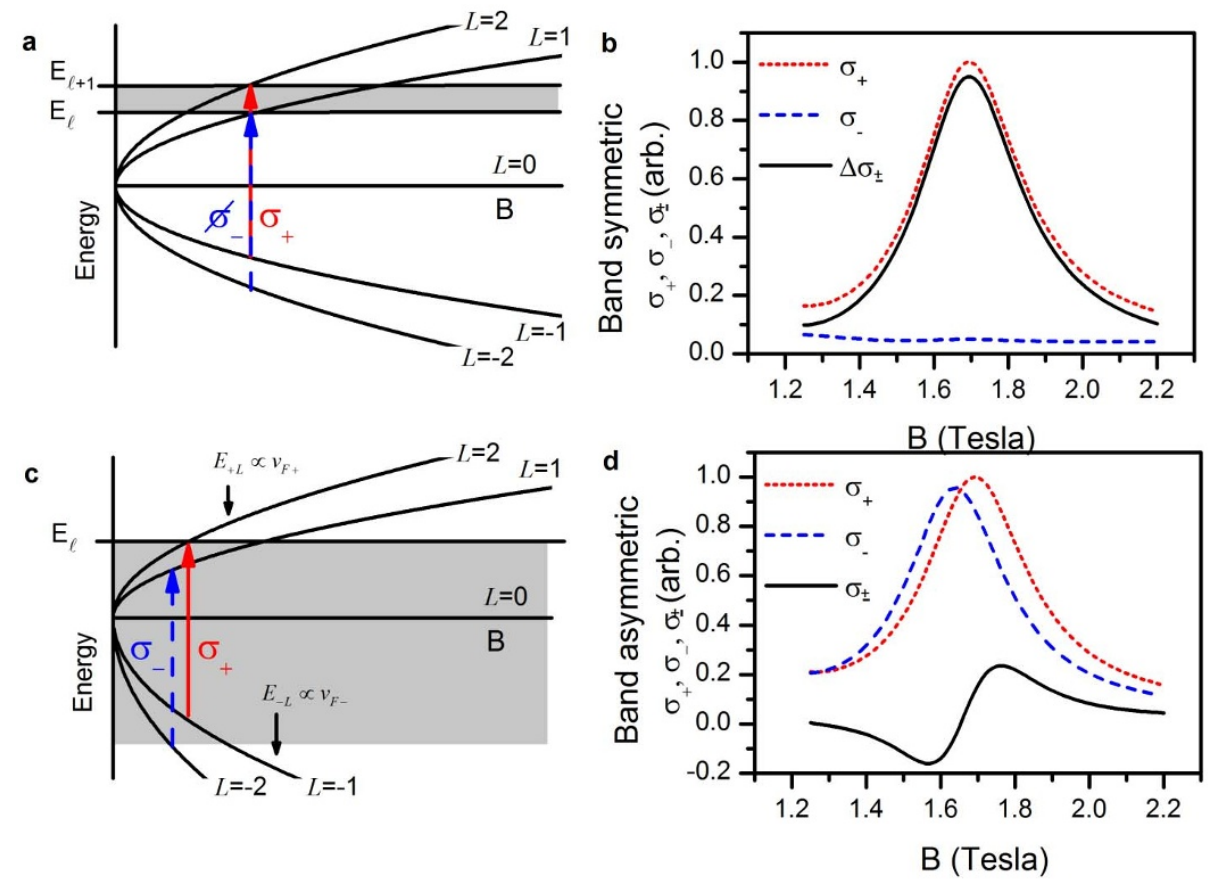

Figure 1 Illustration of chiral symmetry breaking mechanisms caused by: (a,b) Pauli blocking of LLs and (c,d) electron hole band asymmetry. (a) LL energies vs $B$ for monolayer graphene with symmetric electron-hole bands. For this mechanism, optical chiral symmetry is broken when $E_{\ell}<E_{F}<E_{\ell+1}$ (grey shaded region), which results in the Pauli blocking of $-\ell-1 \rightarrow \ell$ LL transitions at CR. (b) Calculation of optical conductivities $\left(\tilde{\sigma}_{+} \neq 0\right.$ and $\tilde{\sigma}_{-} \simeq 0$, red-dotted and blue-dashed curve, respectively) and broken chiral symmetry $\left(\Delta \tilde{\sigma}_{ \pm}=\tilde{\sigma}_{+}-\tilde{\sigma}_{-} \neq 0\right.$, black curve) for Pauli-blocked, band-symmetric graphene. (c) LL energies vs $B$ for monolayer graphene with an electron-hole band asymmetry $\left(\left|E_{L}\right| \neq\left|E_{-L}\right|\right)$. For this mechanism, optical chiral symmetry is broken by lifting the degeneracy between $-\ell-1 \rightarrow \ell$ and $-\ell \rightarrow \ell+1$ transitions, which occurs for a broad range of Fermi energies ( $-E_{\ell}<E_{F}<E_{\ell}$, grey shaded region). (d) Calculation of the optical conductivities $\left(\tilde{\sigma}_{+} \neq 0\right.$ and $\tilde{\sigma}_{-} \neq 0$, red-dotted and blue-dashed curve, respectively) and broken chiral symmetry $\left(\Delta \tilde{\sigma}_{ \pm}=\tilde{\sigma}_{+}-\tilde{\sigma}_{-} \neq 0\right.$, black curve) for graphene with an electron-hole band asymmetry.

In this Report, we use graphene multilayers that vary in thickness and stacking geometry to experimentally investigate how interlayer coupling affects graphene's magneto-electronic structure. We achieve this by using magneto-optical, polarization-sensitive, midinfrared (MIR, 110-224 meV) spectroscopy to study how CRs in multilayer graphene evolve with magnetic field strength $(B)$, photon energy $\left(E_{p h}\right)$, and temperature $(T)$. From these measurements we: 1 ) experimentally demonstrate the validity of theoretical work outlining the LL structure of monolayer ${ }^{16}$, ABA Bernal bilayer and trilayer ${ }^{17}$, and $\mathrm{ABC}$ rhombohedral trilayer graphene ${ }^{18} ; 2$ ) reveal an anomalous temperature dependence of monolayer CRs with implications for broadband, room-temperature optoelectronic devices; and 3) find no detectable band asymmetry in our samples. The last point is of particular interest since our samples are epitaxially grown and require no post-processing for our measurements; thus, the absence of asymmetry strongly suggests that band asymmetry is not intrinsic to graphene. Rather, processing techniques may play a role in enhancing band asymmetry. Furthermore, our measurements indicate that the large polarization response that we observe is activated by Pauli blocking certain LL transitions. This effect opens the door to new high-speed devices that can control the polarization of infrared light through electrostatic gating, even at elevated temperatures.

The polar magneto-optical Kerr effect (MOKE) is the change in light polarization upon reflection from a sample placed in an out-ofplane magnetic field (see Methods section for experimental setup). Changes in the polarization are characterized by the complex Kerr angle $\tilde{\theta}_{K}$, where the real and imaginary components are proportional to changes in orientation and ellipticity of the reflected polarization, respectively. In contrast to conventional infrared transmission/ reflection measurements that probe the average response of the sample to left and right circularly polarized light, the MOKE signal is proportional to the difference in response for opposite handedness of circularly polarized light (i.e., $\tilde{\theta}_{K} \propto \Delta \tilde{\sigma}_{ \pm}=\tilde{\sigma}_{+}-\tilde{\sigma}_{-}$, where $\tilde{\sigma}_{+}$and $\tilde{\sigma}_{-}$are the complex optical conductivities for left and right circularly polarized light, respectively), making it especially sensitive to optical chiral asymmetry $\left(\tilde{\sigma}_{+} \neq \tilde{\sigma}_{-}\right.$or $\left.\Delta \tilde{\sigma}_{+} \neq 0\right)$.

To discuss the expected Kerr effect for graphene, we first consider the simple case of a single monolayer with symmetric electron-hole bands. When applying an out-of-sample-plane magnetic field to graphene, the electronic states condense into discrete energy LLs. For monolayer graphene the LL energies are defined as

$$
E_{L}=\operatorname{sign}(L) \cdot v_{F} \sqrt{2 e \hbar|B L|},
$$

where $v_{F}$ is the Fermi velocity, $L$ is an integer that enumerates the LLs, $L \geq 0$ and $L \leq 0$ define the conduction (electron-like) and valence (hole-like) band LL energies, respectively, and $B$ is the magnetic field strength. As shown in Figure 1a, when the photon energy $\left(E_{p h}\right)$ satisfies the CR condition $\left(E_{p h}=E_{L^{\prime}}-E_{L}\right)$, graphene's two equalenergy interband LL transitions $-\ell \rightarrow \ell+1$ and $-\ell-1 \rightarrow \ell$ are excited, where $\ell$ is a positive integer that enumerates all possible LL transitions (not to be confused with $L$, which is used to enumerate individual LLs). The two allowed transitions absorb the opposite handedness of circularly polarized light (see Figure 1a). Thus, through MOKE measurements we probe asymmetries that exist between the allowed interband transitions in graphene. Such an asymmetry can be caused by two mechanisms: 1) Pauli blocking the $-\ell-1 \rightarrow \ell$ LL transitions, as illustrated in Figure $1 \mathrm{a}, \mathrm{b}$, which yields $\tilde{\sigma}_{-}=0, \tilde{\sigma}_{+} \neq 0$, and $\tilde{\theta}_{K} \propto \Delta \tilde{\sigma}_{ \pm} \neq 0$ and 2$)$ an electron-hole band asymmetry $\left(E_{L} \neq E_{-L}\right)$, which breaks the LL transition energy degeneracy and shifts the left- and right-handed CRs to different $B$ values, for equal photon energies (Figure 1c, d). As discussed later, graphene multilayers yield a more complicated LL energy spectrum than monolayers; however, the LL optical selection rules $(\Delta L= \pm 1)$ 
and mechanisms that cause CR features to appear in the Kerr angle data are the same.

Our samples consist of graphene that is epitaxially grown on the carbon terminated face of silicon carbide $(\mathrm{C}-\mathrm{SiC})^{19,20}$ (see Methods Section for sample details). These samples are ideally suited for our study since C-SiC graphene films are typically comprised of small domains that widely vary in layer thickness and stacking order. Thus, we can study many forms of graphene that coexist in a single sample. Furthermore, these graphene films are known to have high optical mobilities ${ }^{19,21}\left(200000 \mathrm{~cm}^{2} V^{-1} \mathrm{~s}^{-1}\right)$ that are required in order to observe CR at modest magnetic fields ${ }^{51}$.

\section{Results}

Monolayer and bilayer contributions to the Kerr angle. Figure 2a$c$ shows typical results of Kerr angle measurements performed on multilayer C-SiC graphene. We measure $\tilde{\theta}_{K}$ as a function of $B$, with a fixed photon energy $\left(E_{p h}=114.5-133.8 \mathrm{meV}, 230-250 \mathrm{meV}\right)$ and temperature $(T=15-175 \mathrm{~K})$. The data display two distinct sets of CR transition features. The first set, lying in the region $|B|<2 \mathrm{~T}$ is caused by interband LL transitions in monolayer graphene, as indicated by the square-root $B$ scaling $(\sqrt{B})$ of the CR energies versus $E_{p h}$, shown in Figure $2 \mathrm{~d}$. This monolayer response results from rotational stacking faults that frequently occur in $\mathrm{C}-\mathrm{SiC}$ graphene $e^{22,23}$, which effectively decouple adjacent layers ${ }^{5,24-26}$. The second set of CRs is found throughout the entire magnetic field range; however, the strongest features occur for $|B|>2 \mathrm{~T}$. These CRs are characteristic of LL transitions with energies that vary linearly with $B$ (linear- $B$ ), shown in Figure 2e. This behavior is consistent with expectations for $\mathrm{ABA}^{17}$ and $\mathrm{ABC}^{18}$ multilayers, where significant interlayer coupling results in $\mathrm{LL}$ energies that evolve linearly with $B$. The most prominent CR Kerr features yield changes in the polarization up to $60 \mathrm{mrad}$ at $B=5 \mathrm{~T}$. The large magnitude of these polarization changes is comparable to those observed in transmitted far-infrared (FIR, 10-80 meV) radiation ${ }^{27}$. However, the highly doped graphene layers measured in the FIR work ${ }^{27}$ resulted in a large, classical CR polarization response with
$E_{L} \propto B$. For our work the $\sqrt{B}$ nature of MIR CRs indicates that our multilayers are not in the classical CR regime. We also measured $\tilde{\theta}_{K}$ at elevated temperatures as high as $175 \mathrm{~K}$. Interestingly, the magnitude of the CR Kerr features for mono- and multilayer graphene show strikingly different temperature dependences. As depicted in Figure 2f, g, changes in the magnitude of monolayer Kerr effect CRs with increasing temperature are negligible, while linear- $B$ features undergo a $34 \%$ reduction in their magnitude over the range of 15$175 \mathrm{~K}$. As discussed later in this Report, the nearly constant behavior of monolayer features at high temperatures is not expected.

The long downward arrows in Figure 2a, c mark the three most distinct linear- $B$ features that are associated with LL transitions between the upper valence and lower conduction bands in $\mathrm{AB}$ bilayer graphene $e^{17,28}$. It is surprising that $A B$ bilayers produce such large $C R$ Kerr features since both theory and experiments show that stability is reduced for $\mathrm{AB}$ multilayers in $\mathrm{C}-\mathrm{SiC}^{8,29}$. Previous magneto-optical transmission measurements reveal that $\mathrm{AB}$ bilayers are 10 times more scarce than monolayers in $\mathrm{C}$-SiC graphene samples, as indicated by the relative strengths of strong and weak CR absorption features observed for monolayer and $\mathrm{AB}$ bilayers ${ }^{8}$, respectively. Our magneto-optical reflectivity measurements agree with the previous transmission results, where we see a significantly stronger signal associated with the compositionally dominant graphene monolayer response (see $|B|<2 \mathrm{~T}$ features in Figure 2c). However, our Kerr angle data show the opposite effect, where AB bilayer CR features have a much larger magnitude than features associated with monolayer graphene. This indicates that the magnitude of $\tilde{\theta}_{K}$ does not reflect the relative proportion of the various forms of graphene that coexist in a single sample. Additionally, many of the linear- $B$ CRs that appear in the MOKE measurements do not appear in the magneto-reflectivity, indicating that $\tilde{\theta}_{K}$ is significantly more sensitive to small traces of multilayer graphene.

Multilayer contributions to Kerr angle data. While determining the spectral contributions to $\tilde{\theta}_{K}$ from monolayer and $\mathrm{AB}$ stacked bilayer graphene is straightforward, understanding the cause of the
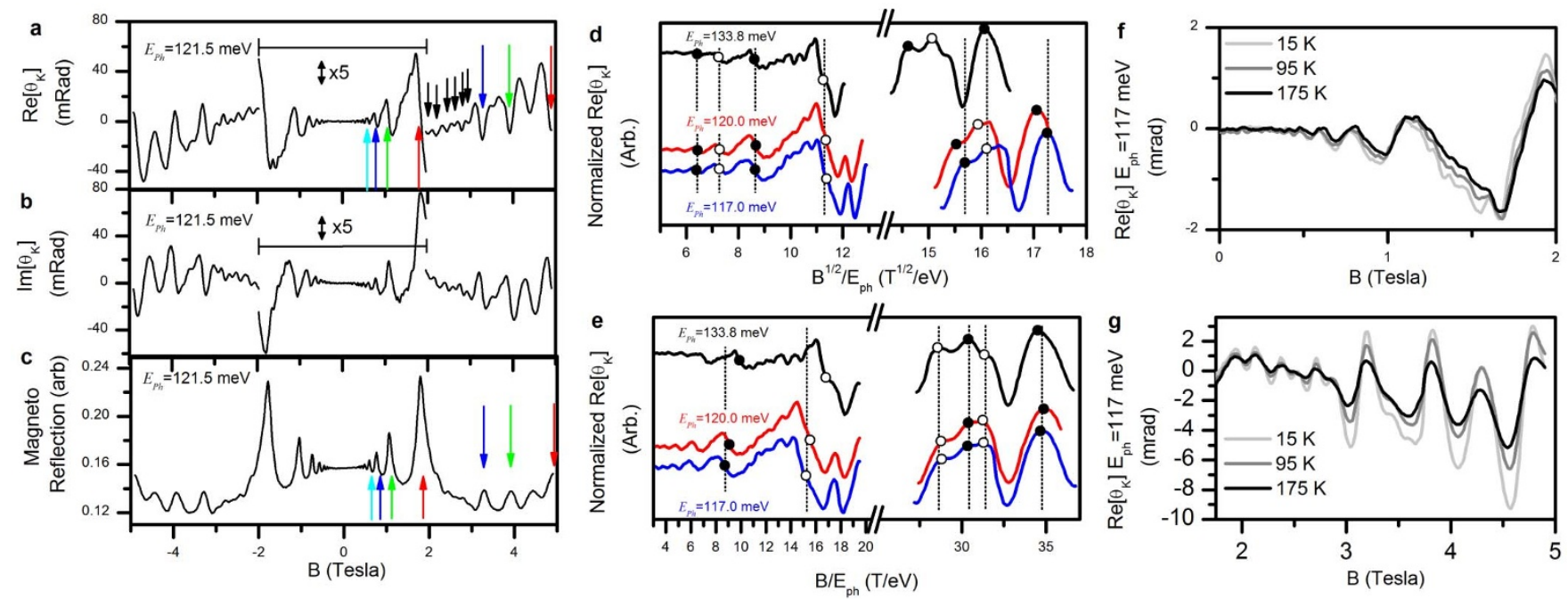

Figure $2 \mid$ General structure of (a) Kerr rotation $\operatorname{Re}\left[\theta_{K}\right]$, (b) Kerr ellipticity $\operatorname{Im}\left[\theta_{K}\right]$, and (c) magneto-reflection for $E_{p h}=121.5 \mathrm{meV}$. The Kerr angles are magnified by a factor of 5 for $|B|<2$ T showing monolayer LL transition features with $E_{\ell} \propto \sqrt{B}$ behavior (up arrows). Structure in the range $|B|>2$ T corresponds to LL transition features with $E_{\ell} \propto B$. Long and short down arrows correspond to features associated with $A B$ bilayer and thicker $\mathrm{ABA} / \mathrm{ABC}$ stacked multilayer graphene, respectively. (d) Kerr data at three photon energies with the horizontal axis rescaled to $\sqrt{B} / E_{p h}$. A vertical alignment of the CRs indicates $\sqrt{B}$ scaling of LL energies. Features at low $B$ clearly show this behavior, suggesting that they correspond to CR in monolayer graphene. (e) Kerr data at three photon energies with horizontal axis rescaled to $B / E_{p h}$. A vertical alignment of the CRs indicates linear- $B$ scaling of $L L$ energies. Features at high $B$ clearly show this behavior suggesting that they correspond to interband LL transitions in $A B A$ and $A B C$ multilayer graphene. (f) Temperature dependence of $\sqrt{B}$ features. Negligible changes occur for temperature ranging from $15-175 \mathrm{~K}$. (g) However, linear- $B$ features decrease by $34 \%$ at $175 \mathrm{~K}$. 
other smaller linear- $B$ features, depicted by the small down arrows in Figure $2 \mathrm{a}$, is not easily accomplished. The increased complexity of these data arises from the large variety of multilayers known to exist in C-face samples ${ }^{29,30}$, each having a unique LL spectrum ${ }^{17,18}$. In particular, ABA stacked graphene with $N$ layers complicates the magneto-optical response, since each multilayer contributes at least N/2 distinct sets of CRs. To simplify and better understand the many contributions to our Kerr angle measurements, we exploit the periodic occurrence of graphene's LL transitions versus inverse magnetic field strength $(1 / B)$. This enables the use of Fourier analysis to reveal CR $1 / B$-frequency components that correspond to each form of multilayer graphene (hereafter, we refer to this technique as Fourier transform Kerr spectroscopy or FTKS). For example, Figure $3 a$ shows the periodic behavior of $\operatorname{Re}\left[\theta_{K}\right]$ for monolayer graphene versus $1 / B$, which is calculated from the complex optical response functions determined by Gusynin et al. $^{16}$. As indicated in Figure $3 \mathrm{~b}, \mathrm{ABA}$ multilayers also produce periodic CR Kerr features versus $1 / B$. These $1 / B$-periods can be detected as $1 / B$-frequencies $(f)$ when applying Fourier analysis to the MOKE signal versus $1 / B$. Figure $3 c$ shows the results of applying this technique to the theoretical Kerr angle response of monolayer graphene, calculated in Figure 3a. Clear peaks are seen at $f$ and higher harmonics $(2 f, 3 f, \ldots)$, where the latter results from the non-sinusoidal-behavior the Kerr/CR response versus $1 / B$. The frequencies detected by FTKS act as a fingerprint for different types of graphene since $f$ is strongly dependent upon the number of layers $N$ and stacking geometry that make up a multilayer (See Supporting Note 1). Thus, FTKS is capable of separating and identifying many different multilayer contributions that coexist in the large probe area of our measurements $\left(9 \mathrm{~mm}^{2}\right)$. Applying the FTKS technique to the measured data dramatically simplifies the signal, as displayed in Figure $3 \mathrm{e}-\mathrm{g}$. In total, seven large $1 / B$ frequency peaks are seen in the FTKS spectrum. These observed 1/ $B$-frequencies are consistent with the theoretical expectations for monolayers as well as $\mathrm{AB}$ and $\mathrm{ABC}$ stacked graphene multilayers.

For $E_{p h}=114.5 \mathrm{meV}$ (Figure 3e) we find multiple peaks corresponding to fundamental and higher harmonic $1 / B$ frequencies that are characteristic of monolayer graphene $\left(f_{\text {mono }}=2.6 \mathrm{~T}, 2 f_{\text {mono }}=5.3 \mathrm{~T}, 3 f_{\text {mono }}=8.0 \mathrm{~T}\right)$. These peaks indicate the presence of rotationally-faulted layers and/or $\mathrm{AB}$ multilayers with odd $N$, where the latter contribution will be discussed later in this Report. At higher $f$, several peaks occur that are consistent with the theoretical expectations for ABA multilayers ${ }^{17}$. The peaks observed at $f_{A B}=18.4 \mathrm{~T}$ and $f_{A B A}=25.8 \mathrm{~T}$ correspond to stacked $\mathrm{AB}$ bilayer and $\mathrm{ABA}$ trilayer graphene, respectively. These $1 / B$ frequencies are in good agreement with theory ${ }^{17}$, where the magnetooptical response of ABA multilayers $(N \geq 2)$ is determined by dividing the system up into sublayers and summing over their individual spectral contributions. For ABA graphene multilayers with an even (odd) number of layers there are $M=N / 2(M=(N+1) / 2)$ sublayers. Thus, a single $\mathrm{AB}$ multilayer with $N \geq 2$ is broken down into $M$ sublayers. Each sublayer contributes a full set of LL
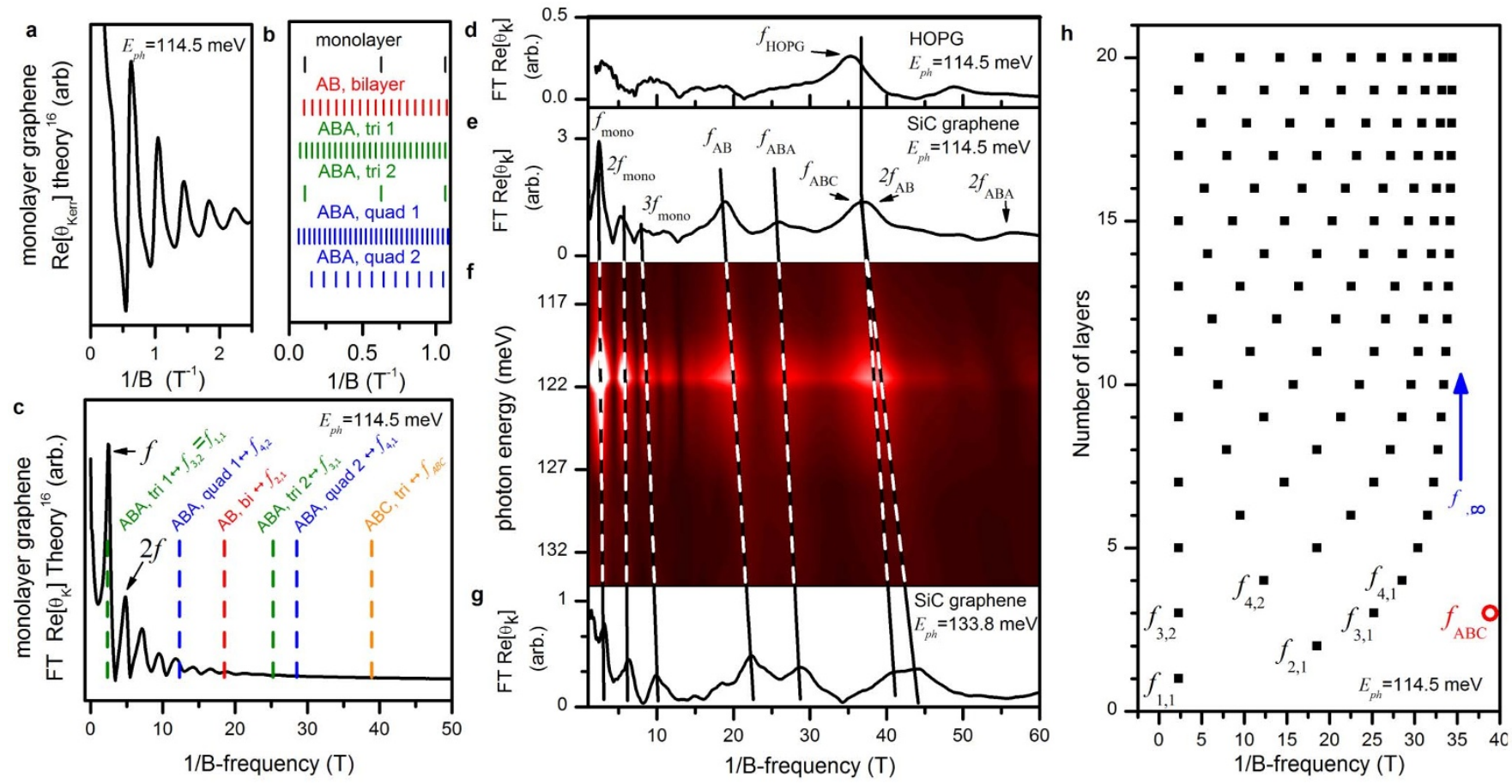

Figure $3 \mid$ (a) Theoretical prediction ${ }^{16}$ of $\operatorname{Re}\left[\tilde{\theta}_{K}\right]$ for monolayer graphene versus $1 / B$, revealing periodic behavior $\left(E_{p h}=114.5 \mathrm{meV}\right)$. (b) Positions of periodic transition features versus $1 / B$ for monolayer and $\mathrm{ABA}$ multilayer graphene, as predicted by Koshino and $\mathrm{Ando} \mathrm{o}^{17}\left(E_{p h}=114.5 \mathrm{meV}, \gamma_{b i}=0.4 \mathrm{eV}\right)$. Note, as predicted by Ref. 17, two sets of periodic transitions for tri- and quadlayer graphene. (c) Fourier transformed Kerr spectrum (FTKS) for monolayer graphene calculated from the curve in Figure $2 \mathrm{a}$, showing a clear fundamental $1 / B$-frequency $\left(f_{\text {mono }}\right)$ and higher harmonics $\left(2 f_{\text {mono }}, 3 f_{\text {mono }}, \ldots\right)$. In addition, the predicted $1 / B$-frequency positions of thicker ABA multilayers are indicated by vertical dashed lines. (d) Measured FTKS spectrum of HOPG for $E_{p h}=114.5 \mathrm{meV}$. (e) $\mathrm{SiC}$ graphene FTKS data for $E_{p h}=114.5 \mathrm{meV}$ marking frequency positions of monolayer $\left(f_{\text {mono }}\right)$, AB bilayer $\left(f_{A B}\right)$, ABA trilayer $\left(f_{A B A}\right)$, ABC trilayer $\left(f_{A B C}\right)$, and their higher harmonics. (f) Photon energy dependence of FTKS. Dark and light areas indicate low and high FTKS magnitudes, respectively. The weakening of the Kerr response away from $E_{p h}=121 \mathrm{meV}$ is associated with the Reststrahlen band of SiC. (g) SiC graphene FTKS data for $E_{p h}=133.8 \mathrm{meV}$ showing multilayer peaks. Note that the $f_{A B C}$ and $2 f_{A B}$ peaks are separated at this photon energy, as expected (see Supporting Note 2 for details). (h) $1 / B$-frequencies $f_{N-m}$ predicted $^{17}$ for the $m^{\text {th }}$ sublayer of ABA multilayers with $N$ layers (black, square symbols). Frequencies are calculated for $E_{p h}=114.5 \mathrm{meV}$ and $\gamma_{b i}=0.4 \mathrm{eV}$. We also show the predicted $1 / B$-frequencies for $\mathrm{ABC}$ trilayer ${ }^{7}\left(f_{A B C}\right.$, red, empty circle) and bulk ABA stacked graphite ${ }^{50}\left(f_{\infty-1}\right.$, blue arrow). 
transitions. Thus, for every multilayer there are $M$ unique sets of CRs and $M$ distinct $1 / B$-frequency components. The LL energies for each sublayer are given by ${ }^{17}$

$$
\begin{aligned}
& E_{N, m, L}=\frac{\operatorname{sign}(L)}{\sqrt{2}}\left[\left(\lambda_{N, m} \gamma_{b i}\right)^{2}+(2 L+1) \Delta_{B}^{2}-.\right. \\
& \left.\sqrt{\left(\lambda_{N, m} \gamma_{b i}\right)^{4}+2(2 L+1)\left(\lambda_{N, m} \gamma_{b i}\right)^{2} \Delta_{B}^{2}+\Delta_{B}^{4}}\right]^{1 / 2}, \\
& \lambda_{N, m}=2 \cos \left(\frac{m \pi}{N+1}\right),
\end{aligned}
$$

where $m$ is an integer enumerating each sublayer, $L$ is the usual LL index, $\lambda_{N, m}$ is a scaling factor, $\gamma_{b i}$ is the interlayer coupling for isolated $\mathrm{AB}$ bilayer graphene, and $\Delta_{B}=v_{F} \sqrt{2 \hbar e|B|}$ is the magnetic energy. In this formulation each sublayer yields LLs that are reminiscent of those expected for an $\mathrm{AB}$ bilayer with a scaled interlayer coupling $\left(\lambda_{N, m} \cdot \gamma_{b i}\right)$. Using equation (2), we numerically determine the unique $1 / B$-frequencies $f_{N, m}$ for each sublayer, as shown in Figure $3 \mathrm{~h}$. In the special case of multilayers with an odd number of AB stacked graphene layers $\left(N_{o}\right)$, the sublayer index $m_{o}=\left(N_{o}+1\right) / 2$ produces $\lambda_{N_{o}, m_{o}}=0$, yielding an effective interlayer coupling of zero and sublayer LLs that are equivalent to monolayer graphene [i.e., equation (2) reduces to equation (1)]. Thus, ABA trilayer graphene will yield both monolayer $f_{\text {mono }}=f_{3,1}$ and bilayer $f_{A B A}=f_{3,2}$ FTKS peaks, as shown in Figure $3 \mathrm{~b}, \mathrm{c}$. The observed $f_{A B A}=f_{3,1} ; f_{A B}=f_{2,1}$ and $f_{\text {mono }}=$ $f_{1,1}$ peaks agree with the theoretical $1 / B$-frequencies that are shown in Figure $3 \mathrm{~h}$. We also observe a peak in the FTKS data near $36.3 \mathrm{~T}$ (labeled as $2 f_{A B}$ and $f_{A B C}$ in Figure $3 \mathrm{e}$ ), which is consistent with predictions for both the second harmonic $\mathrm{AB}$ bilayer and fundamental ABC trilayer components ${ }^{18}$ (see Supporting Note 2 for more details). At higher $E_{p h}$ these peaks separate, as predicted by theory, allowing them to be clearly distinguished (see Figure $3 \mathrm{~g}$ ). To the best of our knowledge, CRs associated with both ABA and ABC trilayer graphene have not been observed in previous studies, making this data set the first to validate the theories presented by References 17 and 18 for thin graphene multilayers. Note that frequencies characterizing thinner multilayers start re-occurring for thicker multilayers (e.g., see Figure $3 \mathrm{~h}$, where $f_{2,1}=f_{5,2}=f_{8,3}$ ). The fact that only a few frequencies are found in our FTKS results suggests that our C$\mathrm{SiC}$ samples are mostly composed of few-layer graphene. For example, an ABA multilayer with $\mathrm{N}=11$ is expected to have six peaks; however, such features are not observed in the FTKS spectrum.

Considering the thick nature of our $\mathrm{C}$-SiC graphene films, one may also expect to find graphitic inclusions in our sample. Theory ${ }^{17}$ predicts a fundamental ABA graphite FTKS peak near $f_{\infty, 1}=35 \mathrm{~T}$ for $E_{p h}=114.5 \mathrm{meV}$ (blue arrow in Figure $3 \mathrm{~h}$ ); however, no such features are observed for our samples. The absence of bulk graphite inclusions is also confirmed by performing FTKS measurements on bulk highly ordered pyrolytic graphite (HOPG). Figure 3d shows the results of these measurements, which confirms the existence of a bulk graphite FTKS peak at $f_{H O P G}=35.3 \mathrm{~T}$. This peak is absent in our measurements on $\mathrm{C}-\mathrm{SiC}$, as seen by comparing Figures $3 \mathrm{~d}$ and $3 \mathrm{e}$, indicating that no graphitic inclusions are present in our multilayer graphene sample.

Band parameter determination. By exploiting the energy dependence of the FTKS features, shown in Figure $3 e-g$, we are able to determine the fundamental band parameters for the multilayers present in the sample (see Supporting Note 1 for fitting details), and further confirm the correct associations of each FTKS component. Fits to $f_{\text {mono }}$ and $f_{A B, b i}$ yield $v_{F}=(1.033 \pm .002) \cdot 10^{6} \mathrm{~m} / \mathrm{s}$ and $\gamma_{b i}=399 \pm 4 \mathrm{meV}$, respectively. Both parameters are consistent with previous work ${ }^{10,21,31}$. Additionally, $f_{A B A}$ and $f_{A B C}$ allow the experimental determination of band parameters for both types of

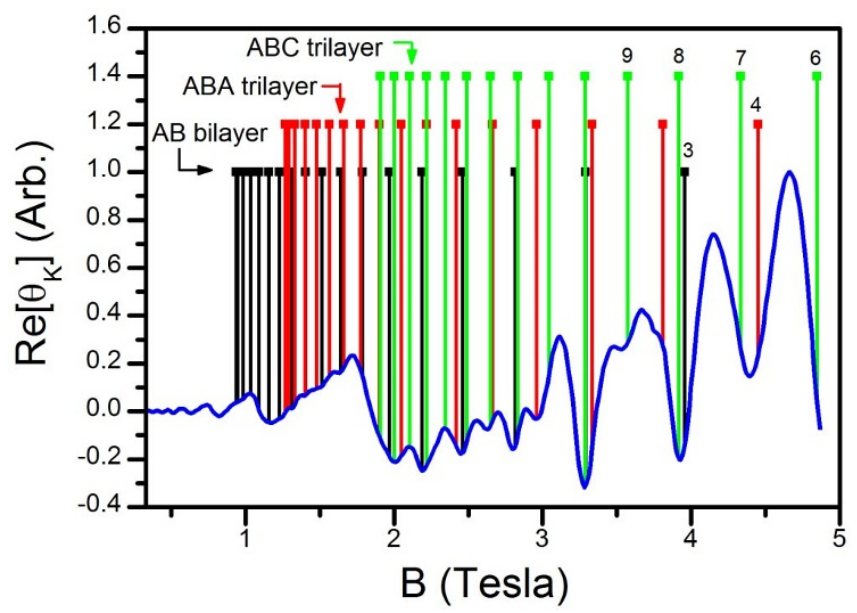

Figure $4 \mid$ Identification of CR features in Kerr angle data vs B. Predicted $\mathrm{CR} B$-values for $\mathrm{AB}$ bilayers (black droplines), ABA trilayers (red droplines), and $\mathrm{ABC}$ trilayers (green droplines). The $\mathrm{CR} B$-values are calculated using the band parameters determined by the $E_{p h}$ dependence of the FTKS data (Fig. 3). The calculation explains the appearance of CR features in the Kerr angle data (blue solid curve). Numeric labels denote the starting LL transition index $(\ell)$ for each type of multilayer graphene. Larger $\ell$ occur at lower magnetic fields.

graphene trilayers. The energy dependence of $f_{A B A}$ yields an effective bilayer interlayer coupling of $\lambda_{3-1} \gamma_{b i}=562 \pm 12 \mathrm{meV}$, which is in excellent agreement with the expected value that is given by equation $2\left(\sqrt{2} \cdot \gamma_{b i}=566 \mathrm{meV}\right)$, further validating the theory of Koshino and Ando ${ }^{17}$. For ABC trilayer graphene we find $\gamma_{A B C}=$ $384 \pm 8 \mathrm{meV}$, which is consistent with previous infrared spectroscopy measurements ${ }^{32}$. As shown in Figure 4, by using these experimentally determined band parameters and LL theory for monolayer, $\mathrm{ABA}$ multilayer, and $\mathrm{ABC}$ multilayer graphene, all major linear- $B$ CR features are accounted for in our $\tilde{\theta}_{K}$ data. The energy-dependent FTKS data also show a dramatic increase in the magnitude of $\tilde{\theta}_{K}$ (Figures $3 \mathrm{e}-\mathrm{g}$ and $5 \mathrm{a}, \mathrm{b}$ ) at $E_{p h}=121 \mathrm{meV}$. The sharp enhancement and sign change of the Kerr features is not due to graphene itself; rather, this effect results from the Reststrahlen band of the $\mathrm{SiC}$ substrate, where the Kerr angle is maximized as the substrate index of refraction approaches unity near $E_{p h} \approx 121 \mathrm{meV}^{33}$.

Band asymmetry. In addition to the band parameters, the symmetry of the conduction and valence bands in graphene is of fundamental interest, since it plays a critical role in shaping the electronic structure. Band asymmetries are widely observed in graphene; however, the mechanism responsible for the band asymmetry depends on the graphene growth technique and the type of sample processing techniques used. In particular, band asymmetries have been attributed to: the twisting of adjacent layers ${ }^{10}$, with asymmetries reaching $14 \%$ for smaller rotations $\left(\theta \sim 3.5^{\circ}\right)$; electrostatic gating of graphene $\mathrm{e}^{12,13}$; and next nearest neighbor interactions (trigonal warping effects) ${ }^{11}$. Band asymmetry may play a key role in producing the optical chiral symmetry breaking (i.e., $\Delta \tilde{\sigma}_{ \pm} \neq 0$, Figure 1c, d) necessary to activate the Kerr angle response. Additionally, changes in the Kerr angle may also be caused by the occupation of $E_{\ell}$ LL states, which Pauli blocks $-\ell-1 \rightarrow \ell$ transitions, as shown in Figure 1a, b. By investigating the $E_{p h}$ dependence of the Kerr angle we are able to put an upper limit on the magnitude of the band asymmetry for the probed layers and determine that the Kerr angle features are primarily caused by the Pauli blocking mechanism.

The band asymmetry for graphene monolayers can be measured quantitatively by comparing FIR intraband CR measurements, 

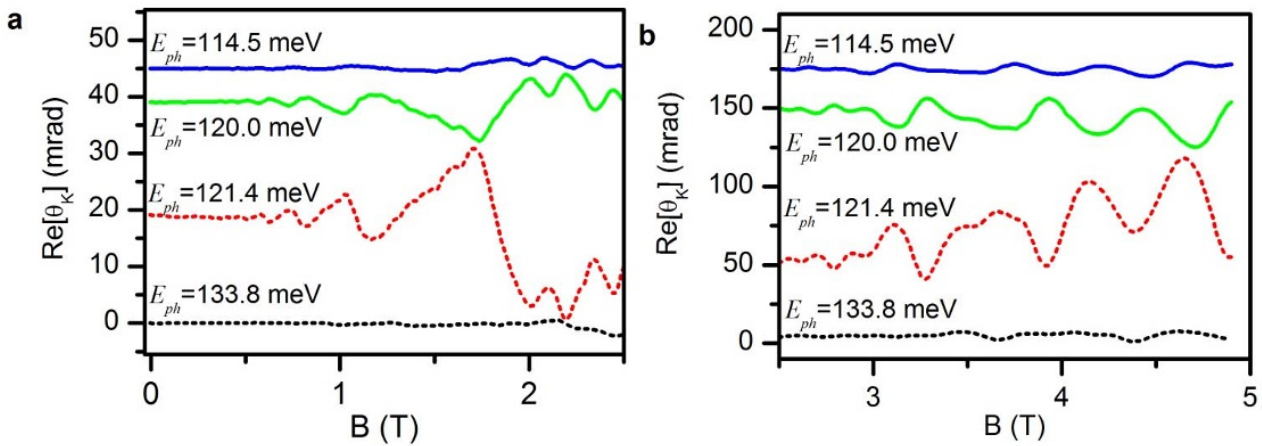

Figure $5 \mid$ (a) $\sqrt{B}$ CR Kerr features for $114.5 \mathrm{meV}<E_{p h}<133.8 \mathrm{meV}$. (b) Linear-B CR Kerr features for $114.5 \mathrm{meV}<E_{p h}<133.8 \mathrm{meV}$. The Reststrahlen band of $\mathrm{SiC}$ is responsible for the sign change of features in solid and dotted curves.

which probe the conduction band shape only, with MIR interband CR measurements that probe the shapes of both conduction and valence bands. Far-infrared measurements of the $0 \rightarrow 1$ intraband CR transition in the same sample enables us to obtain the Fermi velocity $v_{F+}=(1.024 \pm .002) \cdot 10^{6} \mathrm{~m} / \mathrm{s}$ for the conduction band alone $^{34}$. Comparing this to the MIR interband CR transitions, which are related to both the conduction $\left(v_{F+}\right)$ and valence $\left(v_{F-}\right)$ band Fermi velocities, enables us to determine an upper limit of $2 \%$ for the band asymmetry $\left(A=v_{F+} / v_{F-}-1\right)$ of monolayers in our sample. The small band asymmetry is in agreement with previous work ${ }^{34}$ and our fits to the lineshapes of monolayer CR features. For the fitting, we calculate the Kerr angle ${ }^{35}$ from the analytical expressions for the complex optical conductivities of graphene $\left(\tilde{\sigma}_{+}\right.$and $\left.\tilde{\sigma}_{-}\right)$ developed by Gusynin et al. ${ }^{16}$. In general, the CR lineshape is most affected by the choice of Fermi energy and band asymmetry. We find that the best agreement between the MIR Kerr angle data and theory occurs when $E_{F} \simeq 60 \mathrm{meV}$ and $A \simeq 0-2 \%$, as shown in Figure $6 \mathrm{a}, \mathrm{b}$ for $E_{p h}=130.4 \mathrm{meV}$. In order to match the magnitude of CR features in the data and theory, we must employ an overall scaling parameter, which reflects the fact that samples are not uniformly covered by graphene layers that contribute to the measured Kerr response (see Supporting Note 3 ). The excellent agreement between the modeled and measured CR lineshapes indicates that band asymmetry does not significantly contribute to the CR Kerr features observed.

We further investigate the band asymmetry of monolayer graphene by taking advantage of the fact that CR Kerr features caused by band asymmetry are expected to increase in magnitude with increasing $B$ and $E_{p h}$. This effect is attributed to an increase in the $B$-field separation of CR features for $-\ell \rightarrow \ell+1$ and $-\ell-1 \rightarrow \ell$ LL transitions at larger $E_{p h}$, which increases the difference between $\tilde{\sigma}_{+}$ and $\tilde{\sigma}_{-}$. However, at $E_{p h}=224 \mathrm{meV}$, where the band asymmetry and CR Kerr features are expected to be larger (if bands are asymmetric), no features are seen (Figure $6 c, d$ ). Using the parameters determined from the lineshape fitting of the data in the 114$134 \mathrm{meV}$ range, we compare the measurements at $E_{p h}=224 \mathrm{meV}$ with theoretical expectations for the Kerr angle in the presence of
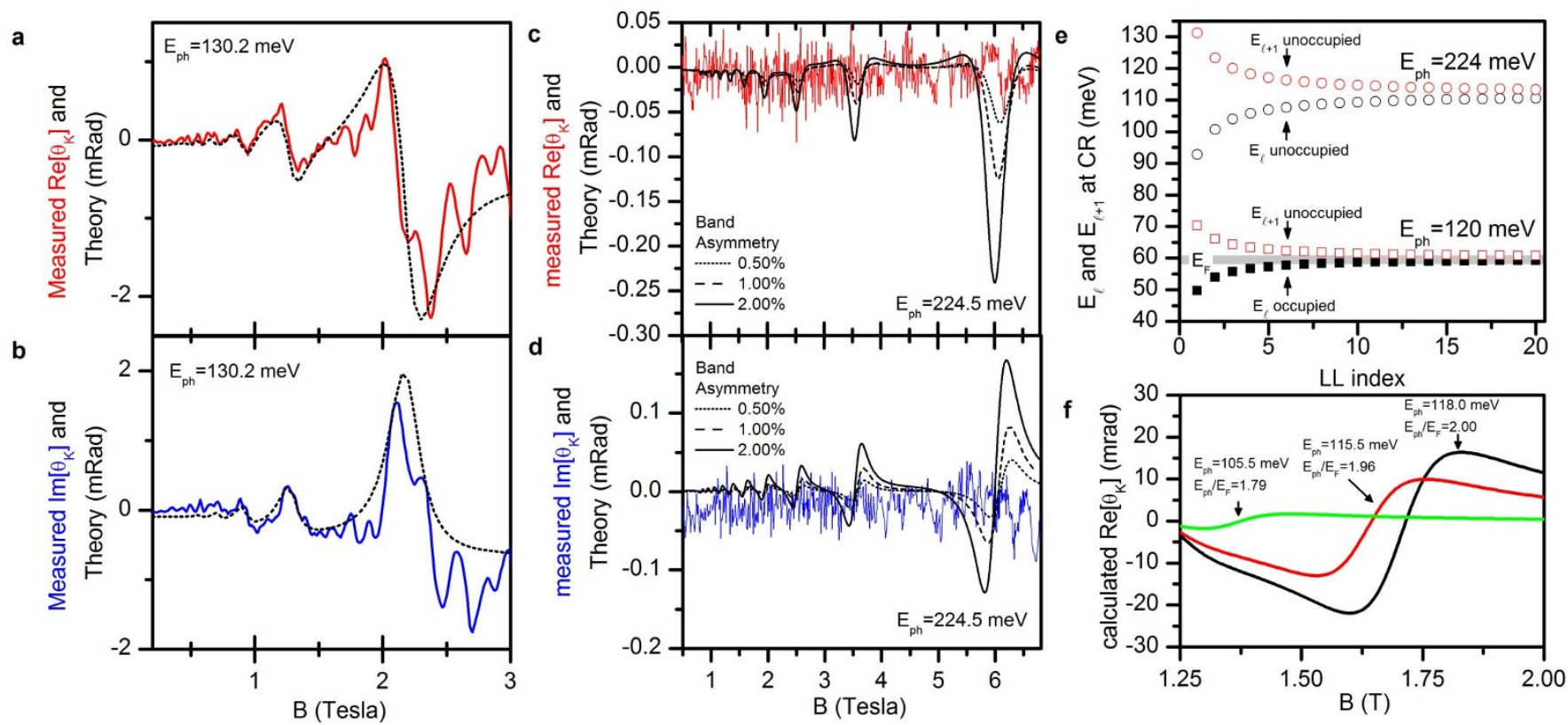

Figure $6 \mid(\mathrm{a}, \mathrm{b})$ Measured (solid, colored curves) complex Kerr angle at $E_{p h}=130 \mathrm{meV}$, which is consistent with theoretical calculation (black, dotted curves) of the complex Kerr response for monolayer graphene systems without electron-hole band asymmetry. (c,d) Absence of CR features in measured complex Kerr angle at $E_{p h}=224 \mathrm{meV}$, which is not consistent with expectations for a band asymmetric graphene system. Black curves are calculations of MIR Kerr response for graphene on SiC with different band asymmetries of $2.00 \%$ (solid curve), $1.0 \%$ (dashed curve), and $0.5 \%$ (dotted curve). The noise of the measurement is sufficiently small to detect the presence of a $0.5 \%$ asymmetry if it were present. (e) Plot of the Fermi energy limits $E_{F, \text { min }}=E_{\ell}=E_{p h}-\Delta_{B} \sqrt{\ell+1}$ and $E_{F, \max }=E_{\ell+1}=E_{p h}-\Delta_{B} \sqrt{\ell}$ for activation of CR Kerr features for $E_{p h}=120 \mathrm{meV}$ and $224 \mathrm{mV}$. At $E_{p h}=224 \mathrm{meV}$ the $E_{\ell}$ and $E_{\ell+1}$ LLs are well above $E_{F}$ and are not occupied, which leads to a null Kerr response since $\tilde{\sigma}_{+}=\tilde{\sigma}_{-}$. (f) Calculation of the weakening of the MIR Kerr angle response in a band symmetric monolayer graphene system for $-1 \rightarrow 2$ CR transition as $E_{p h}$ deviates from $E_{p h, o p t i m a l}=2 E_{F}$. 
band asymmetries ranging from $0.5-2 \%$ (taking into account optical effects of the $\mathrm{SiC}$ substrate). As shown in Figure $6 c$, d, no CR features are observed in measurements to within the noise level, and the magnitude of calculated CR features drops below the measurement noise level for asymmetries less than $0.5 \%$, further suggesting that $\mathrm{CR}$ Kerr features are primarily caused by Pauli-blocked transitions. Although we do not have theoretical models to quantitatively determine the band asymmetry for ordered graphene multilayers, we find that these layers also have symmetric bands, since the $\tilde{\theta}_{K}$ measurements at $E_{p h}=224 \mathrm{meV}$ show no CR features associated with ABA or $\mathrm{ABC}$ graphene multilayers.

This result is an interesting contrast to other works where electron-hole band asymmetries are often detected in exfoliated monolayer flakes on $\mathrm{Si} / \mathrm{SiO}_{2}$ substrates $(2.5 \%)^{36}$ and twisted graphene multilayers grown by chemical vapor deposition (14\% and $8 \%$ for layers with $3.5^{\circ}$ and $21.5^{\circ}$ twisting angles, respectively $)^{10}$. The latter work is of particular interest since it suggests that a large asymmetry could exist for a large fraction of $\mathrm{SiC}$ graphene multilayers with rotational stacking faults, where adjacent graphene layers are typically rotated by approximately $30^{\circ} 24,37$. However, no such asymmetry is observed in our samples, which is consistent with previous studies of unprocessed samples ${ }^{11,14,15}$, suggesting that band asymmetries are strongly enhanced by the chemical transfer process and/or presence of metal contacts or substrates in the vicinity of the graphene areas probed by the measurements ${ }^{12,13}$. Since our measurements show that the band asymmetry in $\mathrm{C}-\mathrm{SiC}$ graphene is less than $0.5 \%$, we suggest that commensurate rotations alone do not cause band asymmetry. Additionally, calculations by Plochocka et $a l .{ }^{11}$ predict asymmetries due to next nearest neighbor hopping in multilayer graphene, where the energy difference between $-\ell-1 \rightarrow \ell$ and $-\ell \rightarrow \ell+1$ transitions increases with field strength as $\Delta \mathrm{E}_{\ell}^{ \pm} \simeq 0.08 B[T] \mathrm{meV}$. Thus, for the $-1 \rightarrow 2$ transition with $E_{p h}=$ $224 \mathrm{meV}$ we expect $\Delta E_{\ell}^{ \pm} \simeq 0.49 \mathrm{meV}$, yielding $1.4 \%$ asymmetry that should make CR Kerr features observable in our measurements. However, these features are not present in our data.

For the band-symmetric Pauli blocking model, CRs appear in the Kerr angle when $E_{\ell}<E_{F}<E_{\ell+1}$ and the CR condition are satisfied simultaneously (illustrated in Figure 1a). As derived in Supporting Note 4, using the monolayer CR condition yields the Pauli blocking condition

$E_{p h} \sqrt{\ell} /(\sqrt{\ell+1}+\sqrt{\ell})<E_{F}<E_{p h} \sqrt{\ell+1} /(\sqrt{\ell+1}+\sqrt{\ell})$,

which indicates that the range of photon energies that yield CR Kerr features is restricted by $E_{F}$. Figure 6e depicts how the limits of equation 3 evolve with the LL transition index $\ell$. At large $\ell$, the energy separation of $E_{\ell}$ and $E_{\ell+1}$ at CR decreases, converging on an optimal photon energy $\left(E_{\text {ph,optimal }}=2 E_{F}\right)$ that Pauli blocks all $-\ell-1 \rightarrow \ell$ transitions, but allows all $-\ell \rightarrow \ell+1$ transitions. Thus, as $E_{p h}$ deviates from the optimal photon energy, the Pauli blocking condition is not met and CR features in the Kerr angle weaken, as shown by theoretical calculations in Figure $6 f$ (multilayers also exhibit this behavior, see Supporting Note 4).

Typically, C-SiC multilayers possess a wide range of Fermi energies. The layers closest to the $\mathrm{SiC}$-graphene interface have the largest $E_{F}^{27,38}$, often exceeding $300 \mathrm{meV}$, which exponentially decays with increasing distance from the substrate ${ }^{39-41}$. The wide range of $E_{F}$ throughout the sample implies that equation 3 will be satisfied for many $E_{p h}$, where graphene layers at different depths (with different $E_{F}$ ) contribute optimally to the CR Kerr response different values of $E_{p h}$. Considering the typical range of $E_{F}$ for C-SiC it is surprising that $\mathrm{CR}$ features are not observed at $E_{p h}=224 \mathrm{meV}$, where graphene layers with $E_{F}=112 \mathrm{meV}$ are expected to contribute to the Kerr response. The absence of features may be explained by the degradation of layer mobility for increasing $E_{F}^{34,41,42}$. It is also possible that the SiC-graphene interface layers, in our sample, have a much lower $E_{F}$ than expected. This was recently observed by Johansson et al. ${ }^{42}$, where $E_{F}$ in substrate interface layers was in the $75-85 \mathrm{meV}$ range.

The observed temperature dependence of monolayer CR Kerr features is especially interesting when considering the band symmetric model. At elevated temperatures near $175 \mathrm{~K}$, the Fermi occupation function broadens and $k_{B} T \approx 15 \mathrm{meV}$ becomes comparable to the LL spacing $\left(E_{\ell+1}-E_{\ell}\right)=5-20 \mathrm{meV}$ for $1-5 \mathrm{~T}$. This reduces the Pauli blocking of $-\ell-1 \rightarrow \ell$ transitions. Thus, it is expected that the magnitude of CR Kerr features will decrease with increasing temperature. As seen in Figure 2g, the magnitude of ABA and $\mathrm{ABC}$ stacked multilayer features behaves as expected. However, monolayer features exhibit a nearly constant magnitude over the range of measured temperatures $15-175 \mathrm{~K}$.

\section{Discussion}

The dominant role of Pauli blocking in producing CR Kerr features in graphene is exciting and holds much promise in the development of future optoelectronic devices. In particular, this work opens the door to devices that can control the polarization of light by tuning: 1) $E_{F}$ away from the ideal value $E_{p h} / 2$ with an electrostatic gate, 2) $E_{p h}$ away from $2 E_{F}$, and 3) $B$ or $E_{p h}$ away from CR. The first point is of particular interest, since an electrostatic gate can modulate $E_{F}$, and, therefore, the polarization of infrared light, very quickly. Such a device is especially important for infrared applications, since current technologies are limited to fixed-frequency, sinusoidal modulation of infrared polarization ${ }^{43}$. In contrast, a graphene-based device is capable of modulating the polarization with arbitrary wave forms at arbitrary frequencies that are an order of magnitude larger than current technologies. Furthermore, comparing the magnitude of measured and theoretical Kerr angles suggests that the maximum changes in polarization, caused by CRs in homogeneous graphene monolayers, may be ten times larger than the angles observed in this work. It may also be possible to further enhance the polarization effects of graphene by exploiting the amplification effect of the Reststrahlen band in $\mathrm{SiC}$ substrates. The realization of a tunable graphene polarizer has the potential to greatly enhance current infrared polarization modulation devices, which are crucial to molecular sensing/identification and also play a role in infrared free space communications.

As demonstrated in this Report, graphene multilayers are capable of producing a large, reflected polarization response (up to $60 \mathrm{mrad}$ ) that is mediated through a combination of CR and optical chiral symmetry breaking. Such behavior is especially evident in highlyheterogeneous, high-quality, large-area graphene that is grown on $\mathrm{C}$-SiC, where we observe over $18 \mathrm{CRs}$ that are attributed to the coexistence of $\mathrm{ABA}, \mathrm{ABC}$, and rotationally faulted graphene multilayers with nearly symmetric electron-hole bands. The excellent agreement between theory and experiment for these multilayers indicates that CR Kerr features are not only predictable, but also highly tunable through the control of layer thickness, stacking order, and the Fermi energy. This understanding of how the magneto-optical properties of graphene evolve from $2 \mathrm{D}$ to $3 \mathrm{D}$ is of fundamental interest to the nanoscience community, where many of the proposed graphene applications aim to exploit a specific trait of a particular type of graphene. Furthermore, the measurement and analysis techniques employed in this Report are able to probe the electronic structure of many types of graphene that may coexist in heterogeneous graphene systems, even if they are stacked on top of each other. Such information can aid in the characterization and optimization of newly proposed devices that are based on graphene heterostructures, such as supercapacitors ${ }^{44}$ and vertical transistors ${ }^{45}$.

\section{Methods}

Sample preparation. In all, four samples are studied. Each sample consists of epitaxial graphene synthesized on nominally on-axis, C-face terminated, semiinsulating $4 \mathrm{H}-\mathrm{SiC}$ by etching the samples in hydrogen and then annealing the 
resulting surface at about $1600^{\circ} \mathrm{C}$ in vacuum $\left(\sim 10^{-4}\right.$ mbar $)$ using a commercial chemical vapor deposition system ${ }^{19}$. The samples consist of a patchwork of multilayer graphene dominated by regions containing up to 50 layers; a small fraction consists of fewer layers, as is confirmed by optical microscopy, micro-Raman spectroscopy, and atomic force microscopy. The $\mathrm{C}$-Face $\mathrm{SiC}$ samples are highly heterogeneous and consist of multilayers with a variety of stacking orders, each having unique electronic properties. The simplest case, AA stacking, consists of neighboring hexagonal layers that have not been shifted in the $2 \mathrm{D}$ plane. $\mathrm{AB}$ multilayers are defined by repeated stacking of A and B layers where B layers are translated along one of the nearest neighbor vectors by exactly one bond length. Similarly, $\mathrm{ABC}$ multilayers, consists of repeated stacking of $\mathrm{A}, \mathrm{B}$, and $\mathrm{C}$ layers, where the $\mathrm{C}$ layer is shifted two bond lengths along the nearest neighbor vector with respect to layer $\mathrm{A}$. In addition, a significant portion of $\mathrm{C}$-face $\mathrm{SiC}$ graphene is known to be commensurate with the $\mathrm{C}$-face $\mathrm{SiC}$ lattice, which leads to rotational stacking faults every 1.6-2.5 layers ${ }^{23}$. While the majority of graphene multilayers on $\mathrm{C}$-face $\mathrm{SiC}$ exhibits stacking faults with adjacent layers rotated by $30^{\circ}$, other rotations have also been observed in smaller quantities ${ }^{22}$. Commensurate stacking arrangements result in an effective decoupling of adjacent layers, which gives rise to their monolayer-like graphene behavior ${ }^{24,26}$.

Measurement system. Measurements are performed on graphene in a magnetooptical cryostat with sample temperatures ranging from 15-200 K and an applied out-of-sample-plane magnetic field up to $7 \mathrm{~T}$. We employ discrete spectral lines from tunable, linearly polarized, mid-infrared (MIR) $\mathrm{CO}_{2}$ (112-138 meV energy, $11-9 \mu \mathrm{m}$ wavelength) and CO (206-248 meV energy, 5-6 $\mu \mathrm{m}$ wavelength) lasers to probe the Kerr effect. This MIR light is directed onto the sample at near normal incidence, and the reflected beam polarization is analyzed using a combination of photoelastic modulation and lock-in techniques that allow for the simultaneous determination of the complex Kerr angle $\left(\operatorname{Re}\left[\theta_{K}\right] \sim\right.$ rotation, $\operatorname{Im}\left[\theta_{K}\right] \sim$ ellipticity) and the magnetoreflectivity ${ }^{35,46-48}$. The probing beam at the sample is on the order of hundreds of microns in diameter and therefore our measurements probe a mixture of response from all forms of graphene present in the laser spot. All measurements consist of positive and negative magnetic field sweeps performed with fixed temperature and probing photon energy.

Inverse magnetic field periodicity. Here we show that monolayer graphene's interband CRs are periodic versus inverse magnetic field. This optical effect is analogous to Shubnikov-de Haas oscillations, which are used in DC magnetotransport measurements to characterize the quantum mechanical nature of $2 \mathrm{D}$ electron systems ${ }^{49}$. The LL energies of monolayer graphene are given by equation (1), where $L<0(L>0)$ defines the valence (conduction) band. Considering an interband transition in graphene, the CR condition yields

$$
E_{p h}=\Delta E_{-\ell, \ell+1}=E_{\ell+1}-E_{-\ell}=v_{F} \sqrt{2 e \hbar B_{T}}(\sqrt{\ell+1}+\sqrt{\ell}),
$$

Where $B_{T}$ is the magnetic field strength at CR. Rearranging equation (4) we find $1 / B_{T}$ is approximately proportional to $\ell / E_{p h}^{2}$ for $\ell>1$. For fixed $E_{p h}$, the nearly linear dependence of $1 / B_{T}$ on $\ell$ implies that $C R s$ are periodic with inverse magnetic field. Similarly, this can also be shown for $\mathrm{AB}$ multilayer graphene and $\mathrm{ABC}$ multilayer graphene by using the LL energies defined by Koshino and Ando ${ }^{17}$ and Yuan et al. ${ }^{18}$ (see Supporting Note 1 for further details).

1. Neto, A. H. C., Guinea, F., Peres, N., Novoselov, K. \& Geim, A. The electronic properties of graphene. Rev. Mod. Phys 81, 109 (2009).

2. Grigorenko, A., Polini, M. \& Novoselov, K. Graphene plasmonics. Nature Photon. 6, 749-758 (2012).

3. Schedin, F. et al. Surface-enhanced Raman spectroscopy of graphene. ACS Nano 4, 5617-5626 (2010).

4. Lee, J., Novoselov, K. S. \& Shin, H. S. Interaction between metal and graphene: dependence on the layer number of graphene. ACS Nano 5, 608-612 (2010).

5. Sadowski, M., Martinez, G., Potemski, M., Berger, C. \& De Heer, W. Landau level spectroscopy of ultrathin graphite layers. Phys. Rev. Lett. 97, 266405 (2006).

6. Jiang, Z. et al. Infrared spectroscopy of Landau levels of graphene. Phys. Rev. Lett. 98, 197403 (2007)

7. Orlita, M. \& Potemski, M. Dirac electronic states in graphene systems: optical spectroscopy studies. Semicond. Sci. Technol. 25, 063001 (2010).

8. Orlita, M. et al. Magneto-optics of bilayer inclusions in multilayered epitaxial graphene on the carbon face of SiC. Phys. Rev. B 83, 125302 (2011).

9. Henriksen, E. et al. Cyclotron resonance in bilayer graphene. Phys. Rev. Lett. 100, 87403 (2008)

10. Luican, A. et al. Single-Layer Behavior and Its Breakdown in Twisted Graphene Layers. Phys. Rev. Lett. 106, 126802 (2011).

11. Plochocka, P. et al. High-energy limit of massless Dirac fermions in multilayer graphene using magneto-optical transmission spectroscopy. Phys. Rev. Lett. 100, 87401 (2008).

12. Huard, B., Stander, N., Sulpizio, J. \& Goldhaber-Gordon, D. Evidence of the role of contacts on the observed electron-hole asymmetry in graphene. Phys. Rev. B 78, 121402 (2008)

13. Malec, C. E. \& Davidović, D. Electronic properties of Au-graphene contacts. Phys. Rev. B 84, 033407 (2011)
14. Orlita, M. et al. Dirac Fermions at the H Point of Graphite: Magnetotransmission Studies. Phys. Rev. Lett. 100, 136403 (2008).

15. Li, G., Luican, A. \& Andrei, E. Y. Scanning Tunneling Spectroscopy of Graphene on Graphite. Phys. Rev. Lett. 102, 176804 (2009).

16. Gusynin, V., Sharapov, S. \& Carbotte, J. Magneto-optical conductivity in graphene. J. Phys: Cond. Mat 19, 026222 (2007).

17. Koshino, M. \& Ando, T. Magneto-optical properties of multilayer graphene. Phys. Rev. B 77, 115313 (2008).

18. Yuan, S., Roldán, R. \& Katsnelson, M. I. Landau level spectrum of ABA-and ABCstacked trilayer graphene. Phys. Rev. B 84, 125455 (2011).

19. Jernigan, G. G. et al. Comparison of Epitaxial Graphene on Si-face and C-face $4 \mathrm{H}$ SiC Formed by Ultrahigh Vacuum and RF Furnace Production. Nano Lett. 9, 2605-2609 (2009).

20. Berger, C. et al. Ultrathin epitaxial graphite: $2 \mathrm{D}$ electron gas properties and a route toward graphene-based nanoelectronics. J. Phys. Chem. B 108, 19912-19916 (2004).

21. Orlita, M. et al. Approaching the Dirac point in high-mobility multilayer epitaxial graphene. Phys. Rev. Lett. 101, 267601 (2008).

22. Hass, J., De Heer, W. \& Conrad, E. The growth and morphology of epitaxial multilayer graphene. J. Phys: Cond. Mat 20, 323202 (2008).

23. Hass, J. et al. Structural properties of the multilayer graphene $4 \mathrm{H}-\mathrm{SiC}(000-1)$ system as determined by surface x-ray diffraction. Phys. Rev. B 75, 214109 (2007)

24. Hass, J. et al. Why Multilayer Graphene on $4 \mathrm{H}-\mathrm{SiC}(000-1)$ Behaves Like a Single Sheet of Graphene. Phys. Rev. Lett. 100, 125504 (2008).

25. Faugeras, C. et al. Few-layer graphene on $\mathrm{SiC}$, pyrolitic graphite, and graphene: A Raman scattering study. Appl. Phys. Lett. 92, 011914 (2008).

26. De Heer, W. A. et al. Epitaxial graphene electronic structure and transport. J. Phys. D: Appl. Phys. 43, 374007 (2010).

27. Crassee, I. et al. Giant Faraday rotation in single-and multilayer graphene. Nature Phys. 7, 48 (2011)

28. Abergel, D. S. L. \& Fal'ko, V. I. Optical and magneto-optical far-infrared properties of bilayer graphene. Phys. Rev. B 75, 155430 (2007)

29. Borysiuk, J., Sołtys, J. \& Piechota, J. Stacking sequence dependence of graphene layers on $\mathrm{SiC}$ (000-1)-Experimental and theoretical investigation. J. Appl. Phys. 109, 093523 (2011)

30. Biedermann, L. B., Bolen, M. L., Capano, M. A., Zemlyanov, D. \& Reifenberger, R. $\mathrm{G}$. Insights into few-layer epitaxial graphene growth on $4 \mathrm{H}-\mathrm{SiC}(000-1)$ substrates from STM studies. Phys. Rev. B 79, 125411 (2009).

31. Li, Z. Q. et al. Band Structure Asymmetry of Bilayer Graphene Revealed by Infrared Spectroscopy. Phys. Rev. Lett. 102, 037403 (2009).

32. Lui, C. H., Li, Z., Mak, K. F., Cappelluti, E. \& Heinz, T. F. Observation of an electrically tunable band gap in trilayer graphene. Nature Phys. 7, 944-947 (2011).

33. Palik, E. D. \& Ghosh, G. Handbook of Optical Constants of Solids. Vol. 3 (Academic press, 1998).

34. Ellis, C. et al. Multi-component response in multilayer graphene revealed through terahertz and infrared magneto-spectroscopy, in the proceedings of the 37th International Conference on Infrared, Millimeter, and Terahertz Waves (2012) http://ieeexplore.ieee.org/xpls/abs_all.jsp?arnumber $=6380102$ (date accessed: 10 / 10/13).

35. Kim, M. H. et al. Determination of the infrared complex magnetoconductivity tensor in itinerant ferromagnets from Faraday and Kerr measurements. Phys. Rev. B 75, 214416 (2007).

36. Deacon, R., Chuang, K. C., Nicholas, R., Novoselov, K. \& Geim, A. Cyclotron resonance study of the electron and hole velocity in graphene monolayers. Phys. Rev. B 76, 081406 (2007)

37. Sprinkle, M. et al. Multilayer epitaxial graphene grown on the $\mathrm{SiC}(000-1)$ surface; structure and electronic properties. J. Phys. D: Appl. Phys. 43, 374006 (2010).

38. First, P. N. et al. Epitaxial graphenes on silicon carbide. MRS Bull. 35, 296-305 (2010).

39. Sun, D. et al. Spectroscopic measurement of interlayer screening in multilayer epitaxial graphene. Phys. Rev. Lett. 104, 136802 (2010).

40. Kuroda, M. A., Tersoff, J. \& Martyna, G. J. Nonlinear Screening in Multilayer Graphene Systems. Phys. Rev. Lett. 106, 116804 (2011).

41. Ohta, T. et al. Interlayer Interaction and Electronic Screening in Multilayer Graphene Investigated with Angle-Resolved Photoemission Spectroscopy. Phys. Rev. Lett. 98, 206802 (2007).

42. Johansson, L. I. et al. Stacking of adjacent graphene layers grown on C-face SiC. Phys. Rev. B 84, 125405 (2011).

43. Oakberg, T. C., Akiyama, T. \& Nakayama, K. A polarization modulator for the far infrared (terhahertz waves). Proc. SPIE 6682, Polarization Science and Remote Sensing III, 66820X, doi:10.1117/12.731827 (2007)

44. Liu, C., Yu, Z., Neff, D., Zhamu, A. \& Jang, B. Z. Graphene-based supercapacitor with an ultrahigh energy density. Nano Lett. 10, 4863-4868 (2010).

45. Britnell, L. et al. Field-Effect Tunneling Transistor Based on Vertical Graphene Heterostructures. Science 335, 947-950 (2012).

46. Cerne, J., Schmadel, D., Rigal, L. \& Drew, H. Measurement of the infrared magneto-optic properties of thin-film metals and high temperature superconductors. Rev. Sci. Instrum. 74, 4755 (2003).

47. Acbas, G. et al. Electronic Structure of Ferromagnetic Semiconductor Ga_ $\{1-\mathrm{x}\}$ $\mathrm{Mn} \_\{\mathrm{x}\}$ As Probed by Subgap Magneto-optical Spectroscopy. Phys. Rev. Lett. 103, 137201 (2009). 
48. Kim, M. H., Kurz, V., Acbas, G., Ellis, C. T. \& Cerne, J. Measurement of the infrared complex Faraday angle in semiconductors and insulators. J. Opt. Soc. Am. B 28, 199-207 (2011).

49. Shubnikov, L. \& de Haas, W. Leiden Commun. 207a (1930). Proc. Netherlands $R$. Acad. Sci. 33, 130-163 (1930)

50. Koshino, M. Interlayer screening effect in graphene multilayers with $A B A$ and ABC stacking. Phys. Rev. B 81, 125304 (2010).

51. Ashcroft, N. W. \& Mermin, N. D. Solid State Physics. Chapters 1 and 14 (Saunders College Publishing, Fort Worth, 1976).

\section{Acknowledgments}

Work done at the University at Buffalo is supported by NSF-DMR1006078. Work at NRL is supported by the Office of Naval Research. J.L.T. acknowledges support from the ASEE. We would like to thank the group of J.P. Carbotte and his post-doc A. Pound for insightful discussions regarding the magneto-optical conductivities of graphene. Many thanks to G. Sambandamurthy and J.L. Musfeldt for suggesting the use of Fourier analysis in our work. We also thank S. Banerjee for the use of his micro-Raman system. Additional thanks to I. Zutic, A.G. Markelz, J. Sinova, J.K. Perron, and A. Mukherjee for manuscript suggestions.

\section{Author contributions}

C.T.E. and J.C. devised the experiment. C.T.E., J.C., and J.G.T. wrote the manuscript. C.T.E., M.H.K., and A.V.S. carried out MIR Kerr measurements. C.T.E. performed analysis and theoretical modeling. J.G.T. and E.R.G. performed preliminary magneto-optical characterization of samples. R.L.M., J.L.T., C.R.E., D.K.G. carried out the growth of multilayer graphene samples.

\section{Additional information}

Supplementary information accompanies this paper at http://www.nature.com/ scientificreports

Competing financial interests: The authors declare no competing financial interests.

How to cite this article: Ellis, C.T. et al. Magneto-optical fingerprints of distinct graphene multilayers using the giant infrared Kerr effect. Sci. Rep. 3, 3143; DOI:10.1038/srep03143 (2013).

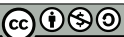

This work is licensed under a Creative Commons Attribution-

NonCommercial-ShareAlike 3.0 Unported license. To view a copy of this license, visit http://creativecommons.org/licenses/by-nc-sa/3.0 